\title{
OUTCOME after spinal-cord injury: influence of specific treatment and prevention modalities
}

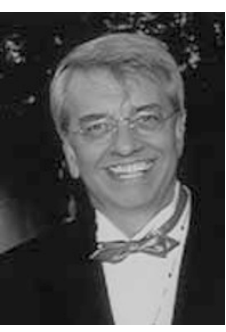

JJ Wyndaele, Editor

Antwerp University Hospital, Antwerp, Belgium

E-mail: spinalcord@uza.be

Dear Spinal Cord reader,

This issue, November 2008, contains several studies on treatment and prevention modalities. Quality of life in people with longstanding spinal-cord injury does depend greatly on healthrelated issues (Lidal et al.). However, health is important for all of us. Several scientific studies have shown that being physically active provides a number of overall health benefits, such as improved quality of life, added energy and a reduction in the risk of serious illness and disease. The importance of eating well and getting good nutrition dates as far back as Hippocrates, who is claimed to have said, 'Let food be your medicine.' Health risks are often highlighted, being overweight, smoking and many more.

When a person becomes paralysed, health-related quality of life differs, as do the health risks. In most of these aspects, specific techniques are being developed for treatment and prevention. This is a never-ending process, which keeps improving care. Several studies such as the use of tracheal stoma stents as a patient-friendly alternative to long-term tracheostomy tubes (Hall and Watt), functional electrical stimulation leg ergometry training (Zbogar et al.), weight-bearing activities (Alekna et al.), a comparison between mechanical ventilation and phrenic nerve stimulation for treatment of spinal-cord injury induced respiratory insufficiency (Hirschfeld et al.), vestibular stimulation to relieve central pain of spinal origin (McGeoch and Ramachandran) and intense exercise in chronic spinal-cord injury (Harness et al.) are published in this issue.

Venous gas bubble formation and decompression are known as one of the possible causes of spinal-cord lesion. A study by Breskovic et al. investigates the risk after scuba diving in persons with chronic spinal-cord injury.

When conducting epidemiological and health services research, registers can be one obvious way to collect data. A Swedish study looked into the limitation of using a register without any validation process and into a way to avoid underestimations (Nordgren).

An animal study is presented in which the question of whether intrathecal papaverine induces changes in spinal cord blood flow of the injured spinal cord and may prevent secondary injury is investigated (Yong et al.).

Three interesting case reports have been included in this issue.

Spinal Cord (2008) 46, 709; doi:10.1038/sc.2008.130 